\title{
Traceability in Healthcare: Crossing Boundaries
}

C. Lovis

University hospitals of Geneva, Service of Medical informatics, Geneva, Switzerland

Chair of the European Federation of Medical Informatics Working Group on Traceability

\section{Summary}

Objectives: This paper is a survey on the problem of traceability in healthcare. Traceability covers many different aspects and its understanding varies among different players. In supply chains and retails, traceability usually covers aspects pertaining to logistics. The challenge is to keep trace of objects manufactured, to track their locations in a production and distribution processes. In food industry, traceability has received a lot of attention because of public health problems related to infectious diseases. For instance, in Europe, the challenge of traceability has been to build the tracking of meat, from the living animal to the shell. In the health sector, traceability has mostly been involved in patient safety around human products such as blood derivates contaminants or implanted devices and prosthesis such as mammary implants. There are growing interests involving traceability in health related to drug safety, including the problem of counterfeited drugs, and to privacy. Traceability is also increasingly seen as a mean to improve efficiency of the logistics of care and a way to better understand costs and usage of resources.

Methods: This survey is reviewing the literature and proposes a discussion based on the real use and needs of traceability in a large teaching hospital.

Results and Conclusion: Traceabilityin healthcare is at the crossroads of numerous needs. It is therefore of particular complexity and raises many new challenges. Identification management and entity tracking, from serialization of consumers' good production in the supply chains, to the identification of actors, patients, care providers, locations and processes is a huge effort, tacking economical, political, ethical and technical challenges. New requirements are needed, not usually met in the supply chain, such as serialization and persistence in time. New problems arise, such as privacy and legal frameworks. There are growing needs to increase traceability for drug products, related to drug safety, counterfeited drugs, and to privacy. Technical problems around reliability, robustness and efficiency of carriers are still to be resolved. There is a lot at stakes. Traceability is a major aspect of the future in healthcare and requires the attention of the community of medical informatics.

\section{Keywords}

Traceability, identification management, logistics, supply chain, EPC, GST

Geissbuhler A, Kulikowski C, editors. IMIA Yearbook of Medical Informatic 2008. Methods Inf Med 2008; 47 Supp 1 :105-13

\section{Introduction}

Traceability is a well-known aspect in numerous industries since decades. In logistics, traceability refers to the capability for tracing goods along the distribution chain, from the suppliers to the retailers. In other activities, traceability will include the production chain, together with the unique identification of all raw products and items needed to create or build the final good, as well as all steps involved in the production processes. For example, traceability is an important aspect in the automotive industry, where it makes recalls possible, or in the food industry where it contributes to food safety [13]. Food safety itself has become a scientific discipline describing the handling, preparation, and storage of food in ways that prevent food borne illness. Beside the formalization and description of numerous routines important to respect in order to avoid potentially severe health hazards, traceability in food industry is also of major importance to allow the identification of the origin of feed and food ingredients. Food sources are of prime importance for the protection of consumers, particularly when products are faulty, and the identification of the source of the problem is a health priority. Traceability facilitates the withdrawal of foods and enables giving consumers' targeted and accurate information concerning implicated products. Food safety is a good model to understand the needs and goals of traceability in healthcare. Traceability can be defined as the abil- ity to interrelate the uniquely identifiable entities in a way that matters. Finally, it is important to mention that traceability can have serious impacts on the public, as illustrated with food traceability, which has been, in Europe, one of the most important tools used to restore confidence in meat originated from beef after the cow's disease scandal $[4,5]$. The importance of traceability related to the public's opinion and confidence in healthcare must not be underestimated, especially when it comes to privacy and safety [6].

Traceability and identification management techniques applied to the healthcare sector receive a growing attention, both from the industry and from the scientific community.

The increasing interest for traceability in healthcare is well emphasized in figure 1 that shows the number of yearly publications indexed in Medline and that can be found with the query traceability[All Fields]. This figures shows also that traceability is a young and still small field in the healthcare domain, with less than 500 publications as of December 2007. When looking at these publications in more detail, most of them relate to laboratory measures [7], donor's banks and blood products [8-10] or environmental menaces. A few only are devoted to non-human tissues or goods traceability, such as drugs, implants or devices; almost none address the problem of supply chain traceability in healthcare and logistics. The very recent creation of the Traceability working group of the European Federation 
of Medical Informatics (EFMI), launched in 2006, while there is still no working group devoted to this topic at the American Medical informatics Association (AMIA) or at the International Medical Informatics Association (IMIA) emphasizes the need to put more attention on this field. Noticeably, the repartition of the publications on the topic is not so well distributed in countries, with five leading countries, USA, England, Germany, France and the Netherlands accounting for about $90 \%$ of all publications (419 of 476).

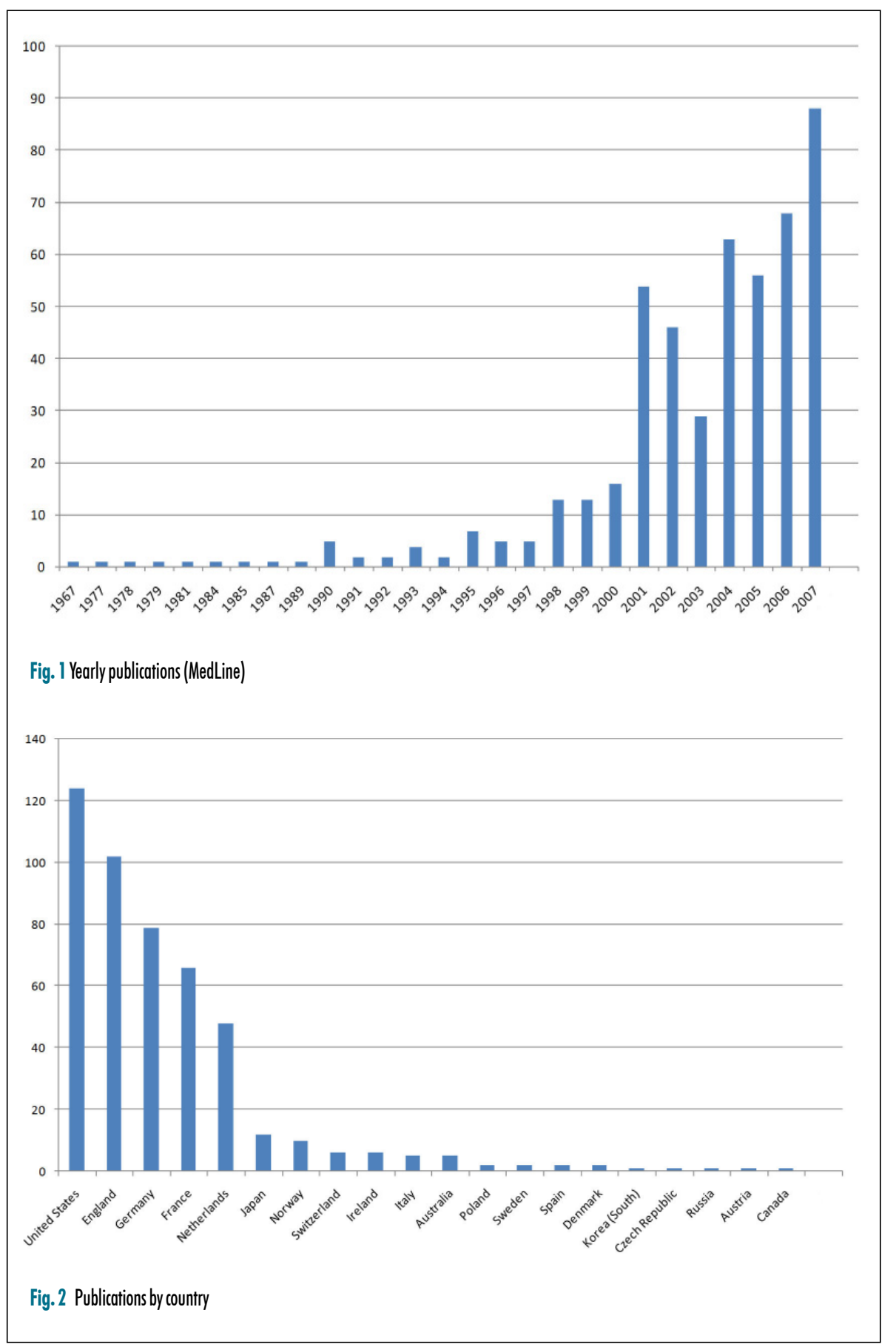

Traceability is an important domain of healthcare, and is devoted to become a major discipline of information technologies in this field. Comparably to food safety, public health and individual safety require a growing ability to track faulty products. However, traceability is much more than that, especially in the complex health world, embedded in a very rich supply chain and logistics networks.

\section{Place of Traceability in the Healthcare System}

There are many benefits to expect from track and trace management in the healthcare sector. Among them, it is worth to emphasize

Improved supply chain efficiency
Prevention of errors
Public health
Medico-legal investigations
Clinical research and epidemiological surveillance
Flows and processes management
Billing
Counterfeit measures

Table 1 Expected benefits

\section{Improved Supply Chain Efficiency}

Care providers, patients and healthcare facilities are at the crossing roads of many supply industries: pharmaceutical industries, medical devices, disposable products, implantable devices to quote the most evident, but also numerous other suppliers, such as food and groceries, beds, clothes, computers, office equipments, etc. Many hospitals are comparable to small cities, with distributed stocks management and internal markets, embedded in the global market, in a global world. Improving 
this management through greater visibility, accuracy and velocity is expected to produce to the same improvement in economic efficiency as measured in other industries.

Traceability is a crucial component of logistics, which is the optimal management of flows and resources. Logistics includes physical flows, such as buildings, materials, objects, people; logical flows such as information or processes descriptions and finally financial flows.

\section{Prevention of Errors}

Numerous errors in medicine are directly or indirectly related to entities identity management. Errors due to erroneous patient identification or bad drug identification are far from rare. Unique identification of patients and care providers as well as encoding of the drug dose units or the packages units of use to enable automated verification, therefore enforcing the verification of the right dose, for the right patient at the right time, would be of great help in this domain.

\section{Public Health}

Public health has important needs related to the ability to track and trace back population-based events to their causal roots. A complete track and trace mechanism from the manufacturer to the patient would be very helpful, especially when faulty products are detected. These needs are illustrated in many aspects, such as tracing all patients having received a specific drug, or prosthesis, any kind of material, drugs, immunizations, human products that are used with patients. Unfortunately, the availability of traceability is very heterogeneous. For some type of items, very good traceability is avail- able, such as pacemakers, for others, such as drugs, it is almost non-existent. In addition, the legal framework enforcing traceability is very dependent from national or state level policies. Therefore, a global approach would be of highest value, as already proposed for food [11].

\section{Medico-legal investigations}

The use of an interoperable framework of identification schemes allowing the identification of actors, objects, locations and actions in a temporal net of events is priceless for medico-legal investigations, facilitating the "reverse engineering" of the cascade of events leading to unwanted or unexpected incident, or allowing to clarify the use of the patient records when privacy breaches are suspected.

\section{Clinical Research and Epidemiological Surveillance}

The high cost of clinical research, for example in the domain of drugs sideeffects surveillance, would benefit highly by moving from the current situation, with surveys limited in time and populations towards continuous monitoring. A robust track and trace management system would be of great help, leveraging the power of secondary usage of clinical data.

\section{Flows and Processes Management}

One of the biggest challenges faced by healthcare systems is around improving global care management. This includes clinical pathways in hospitals, community networks, continuum of care between in- and out-patients, all along the life of patients, extending towards wellness and citizens. However, this challenge is tightly linked to the ability to build interoperability between all systems.

\section{Billing}

Billing is certainly the domain of healthcare best developed using information technologies. However, a lot of work is still done by hand. Availability of a robust actors and asset identification and management system is mandatory in this field.

\section{Counterfeit Measures}

Counterfeited drugs are increasingly available in the world. While such drugs were only available on specific markets segments, they are extending rapidly in most markets due to the use of internet. Counterfeited drugs cause important financial losses to pharmaceutical companies, and are the direct cause of increased morbidity and mortality in the world. One of the best weapon against counterfeited drugs is a complete track and trace management systems able to authenticate drugs and their origins. Several initiatives are ongoing to help reducing counterfeited drugs, such as the use of ePedigree and serialization [11].

The list of benefits in Table 1 is not exhaustive. However, traceability could and should be much more developed in the healthcare sector. As shown in the first two figures, traceability has only recently come to the attention of the scientific medical community, with few important exceptions such as laboratory and blood products. The same remark applies to the medical informatics community, where there are only rare papers about implementation of traceability in the patient record [1215]. One of the explanations is probably linked to the fact that traceability 
has only recently gained attention of the medical informatics community is because the field of traceability is usually associated with logistics and supply chains. Therefore, traceability relates historically more to ordering, stocks and assets management and, therefore, more in charge of the IT department. However, with the raising attention given to patient safety, to prevention of errors and the need for improved efficiency in care processes, clinical information systems are receiving more and more focus and are increasingly put at the center of the healthcare industry. As such, the trend is to consider clinical information systems to be at the heart of the health information systems, embedded and surrounded with the logistics on one side, allowing the provision of care, the financing on the other side, with billing and recovery and finally the data management on the last side. The data management is related to secondary use of data, covering governance, policymaking, public health, clinical research, etc. In this vision, traceability along with track and trace mechanisms, can be seen as a folding activity requiring to build a deep network of links between physical or virtual objects, actors, locations or actions. Drug traceability could be a good example to illustrate this. The pharmaceutical industry does encode worldwide all production batches with GS1 identifications (see below). These numbers allow identifying both the company, the drug and the production batch of the drug, a sort of mix between classification (drug) and instantiation (company and batch). For some drugs, also a serialized GS1 Global Trade Item Number (GTIN) will uniquely identify each orderable item of that drug. Ordering mechanism from the user, hospital, care-providing facilities, pharmacy, can use the GS1 GTIN to identify the drug to be or- dered. The same GS1 standard can be used to track stocks and locations, validate best-before dates, etc. When releasing the items, these numbers can be scanned at the same way they are at checkout in general stores. In healthcare, the next step should be to track the drug up to the pill and the patient taking it, the nurse or pharmacist distributing it, the physician ordering it. In order to close the loop, it will be mandatory to extend the usage of traceability to all items and actions.

\section{GS1: An Existing Solution for Traceability}

The GS1 organization was formed in early 2005 from the joining of EAN International and the Uniform Code Council (UCC). GS1 is a folder organization grouping more than $100 \mathrm{mem}$ -

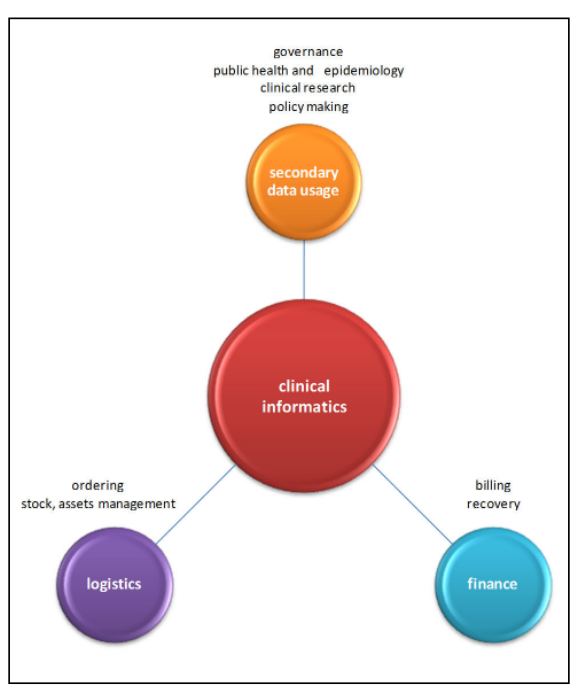

Fig. 3 Traceability requires to cross IT boundaries ber organizations, representing all points in the supply chain, has over a million companies doing business across 145 countries, over 20 represented sectors (FMCG, healthcare, transport, customs, etc.), and operates more than 5 billion transactions a day. GS1 is a family of standards developed since 30 years in order to improve the efficiency of the supply and the demand chains. The GS1 standard is either ISO standards (unique identifications. Data carriers), part of UN/CEFACT (messaging, classification) or ITU (radio frequencies) frameworks. In short, GS1 covers standards for automatic identification of assets, items and locations; standards to improve electronic business messaging, for global data synchronization and business transactions and standards on carriers, such as barcodes and RFID. A very important point about the GS1 family of standards is that, by the nature of the domain of trading, these standards are used worldwide, across political and economical borders, across companies and activity sectors. A barcode using the GS1 encoding and representation printed on items produced can be read worldwide all along the supply chain, up to the shelf and the final user. This characteristic is becoming crucially important in the healthcare sector with the increased mobility of citizens.

A GS1 encoding is usually seen as an alphanumeric sequence or a barcode. The reality of the GS1 encoding is far more complex and a complete discussion of the GS1 anatomy, physiology and limitations goes far beyond the purpose of this paper. The GS1 model supports several main groups, such as entities, iden- 


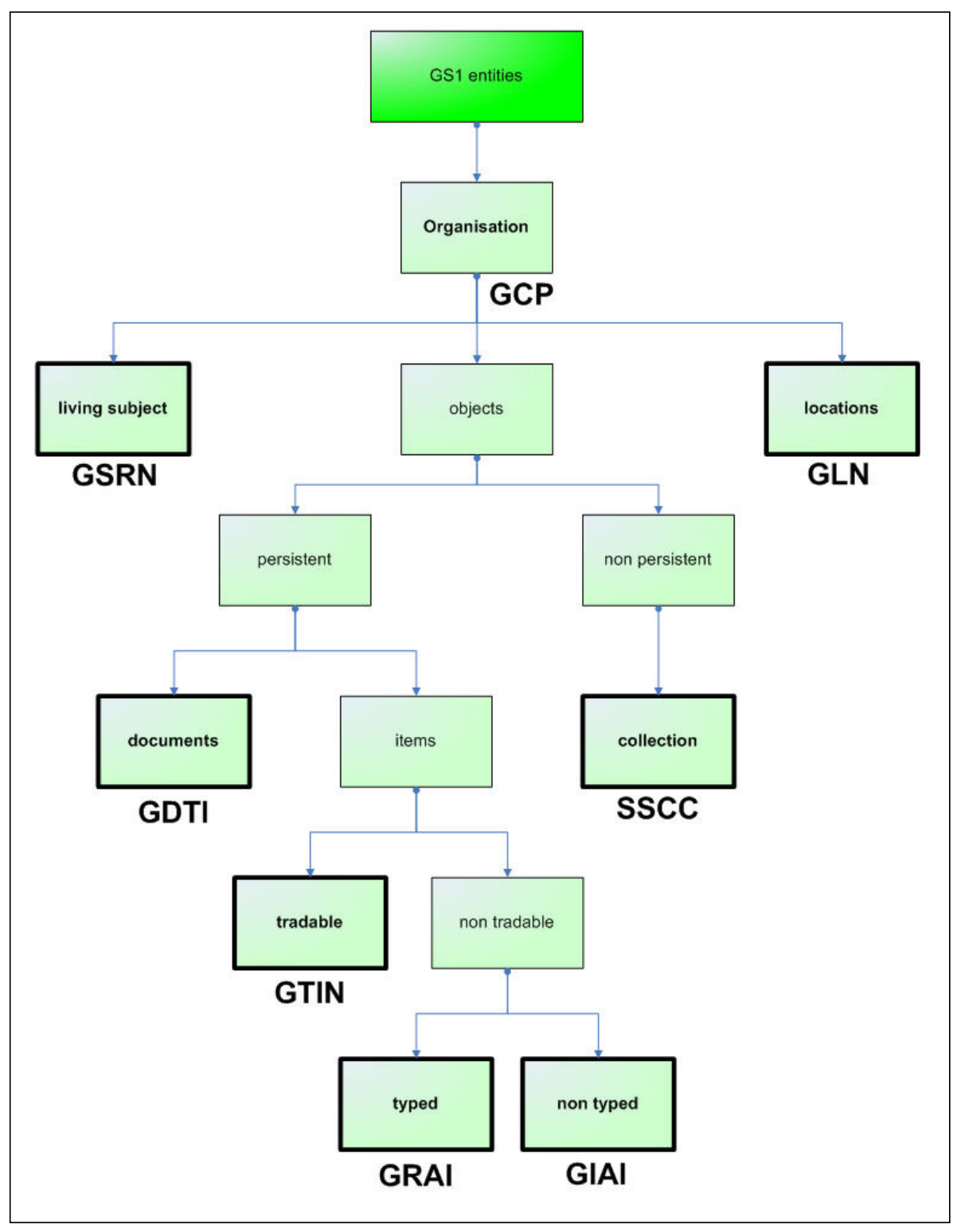

Fig. 5 GSI - Model of entities

Table 2 Some Applications Identifiers (Al)

\begin{tabular}{|l|l|l|}
\hline Al & Description & digits \\
00 & SSCS (Serial Shipping Container Code) & $\mathrm{n} 18$ \\
01 (and 02) & GTIN (Global Trade Item Number) & $\mathrm{n} 14$ \\
$410-415$ & GLN (Global Location Number) & $\mathrm{n} 13$ \\
253 & GDTI (Global Document Type Identifier) & $\mathrm{n} \ldots 30$ \\
8018 & GSRN (Global Service Relationship Number) & $\mathrm{n} 18$ \\
8003 & GRAI (Global Returnable Asset ID) & an...30 \\
8004 & GIAI (Global Inventory Asset ID) & an...30 \\
\hline
\end{tabular}

tification, serialization, locations, transactions, etc. An attempt of representation of the entity model found in GS1 is represented in Figure 5 (see [16] section 5.4 page 231 for more information).

The usual elements of GS1, such as the GCP (Global Company Prefix) uniquely identifying the company or system user, or the GLN (Global Location Number) can be represented in a multiaxial and combinatorial model. These elements are usually referred as sequential information in the encoding found on tradable items, such as drugs and other medical devices. For most items, the identification is made by the mean of a GTIN, the Global Trade item Number. The Global Trade Item Number (GTIN) is used for the unique identification of trade items worldwide. A trade item is any item, including products or services, upon which there is a need to retrieve pre-defined information and that may be priced, ordered or invoiced at any point in any supply chain. This includes individual items as well as all their different configurations in different types of packaging. These data structures provide unique identification when they are right justified in a 14-digit reference field. GTIN's are based on the GCP, the Global Company Prefix, so they allow identifying both the item and the brand owner.

The global adoption of GS1 throughout the complete healthcare system would have huge advantages, complying with an existing system widely used in manufacturer and supply chains and that proved already to be efficient for processes management. However, there are problems to be solved in the GS1 system. For example, the GLN is used to identify both a function and a precise location, such a (any) refrigerated place or this very precise location, mixing classes and instances. This problem should be addressed with serialization, allowing room to identify each specific instance of a class of entities. 


\section{Limitations and Challenges}

The limitations of the accessibility of information are recognized as an important cause of medical errors and loss of efficiency in the healthcare sector [17-21] and there are important initiatives worldwide to promote interoperability and improved availability of information in the healthcare sector. However, these numerous initiatives are facing major obstacles.

\section{Table 3 Some limitations and challenges}

Privacy, legal and ethical issues

National, state level policies

Actors identification

Facilities unique identification

Serialization and items unique identification

Ontologies and information models

Persistence in time

Carriers and readers

\section{Privacy, Legal and Ethical Issues}

The most important goal to be achieved by traceability is, by nature, a serious breach to citizen privacy and is getting in contradiction with most existing legal and ethical frameworks. Traceability should allow tracking and tracing all actions, items, actors involved in the process of requiring, deciding, organizing and providing care, including short- and long-term outcomes. It could result in creating a complete transparency in the healthcare system, which is by far not acceptable towards citizen's privacy, citizens as patients or care providers. The best solutions to these contradictory needs are not yet found, but it is clear that just avoiding this problematic is not the right answer. The traceability network is slowly growing due to the supply chain and billing needs without clear rules and policies, a situation that might become inextricable in the near future.

\section{National, State Level Policies}

An interesting way of comparing healthcare and trading businesses is to compare the roles and actions of the World Health Organization and the World Trade Organization. The World Trade Organization (WTO) defines itself as the only global international organization dealing with the rules of trade between nations. At its heart are the WTO agreements, negotiated and signed by the bulk of the world's trading nations and ratified in their parliaments. The goal is to help producers of goods and services, exporters, and importers conduct their business ${ }^{1}$. The WHO is the directing and coordinating authority for health within the United Nations system. It is responsible for providing leadership on global health matters, shaping the health research agenda, setting norms and standards, articulating evidencebased policy options, providing technical support to countries and monitoring and assessing health trends ${ }^{2}$. These two agencies reflect the major differences in the legal and policies framework existing between trading and healthcare. Policies and rules in healthcare are very heterogeneous, between countries and often between states. There are few policies, if any, recognized and applicable at the international level, one example of such a research being the IMPACT project of WHO to fight counterfeited drugs. This is a clear hindrance

\footnotetext{
http://www.wto.org/english/thewto_e/whatis_e/ whatis_e.htm

2 http://www.who.int/about/en/
}

when it comes to build and create systems such as traceability that should cross such boundaries, or at least be compatible with all these various legal environments.

\section{Actors Identification}

Unique identification of all actors involved, patients, care providers, but also all actors of the supply chain, the logistics, the billing, etc. will end up in identifying uniquely the whole population. Such identification could be specific to each country, though complying with some standards that could be used worldwide, in order to allow continuity of care, and informational chain, cross borders. Switzerland, for instance, allocates a unique GS1 identification to every physician. Because of increased mobility of citizens, patients and care providers, an interoperable international identification would be appropriate, such as the Global Service Relationship Number (GSRN), from the GS1 standard. However, in addition to be technically difficult to realize, such unique identification leads to important political and ethical concerns, not to speak about authentication, which is of increasing worries. Identities usurpation for insurance fraud is already a problem. Therefore, a completely closed system would require both identification and authentication, which is a long-term goal. The current trend is towards interoperable insurance and health professional smartcards, such as in the European Union. These cards provide some identification linked to the insurance coverage and reimbursement facilities. Cost control and billing efficiency, more than patient safety and continuum of care, appear to be important driving forces towards actors identification, which may lead to dangerous over simplifications. 


\section{Facilities Unique Identification}

The ability to identify uniquely, worldwide, all care organizations by using GS1 Global Company Prefixes (GCP) associated with GLN's (Global Location Number) for their facilities, producing a sort of general yellow pages, would be of great help to facilitate the accessibility of information in healthcare. It would help not only the logistic part of suppliers, but also billing, relationships with public bodies and help to reference potential information about patients. Implementations of this kind exist or are in process in Switzerland, United States or Denmark.

\section{Serialization and Items Unique Identification}

An important aspect of traceability of items is related to their unique identification. This is still an aspect that is not well covered by existing systems, even if the GS1 system provides some item serialization. In order to implement traceability, one cannot have only identification at the packaging or batch level. Each single item shall be identified, getting to the serialization of each code. Every single pill, single syringe, or any other item used in healthcare must have a unique identification which could then be used within complete system, from supplier, to the facilities or the retailers, to the patient. Such an encoding is needed to implement, for example, bedside checking when administrating drugs to patients [12]. Serialization is, for now, expected to be reserved for specific drugs or regiments, such as chemotherapies or devices. But, one can wish serialization to be extended to implants all drugs, as the effects on long-term outcomes is hard to predict, as shown in many cases in the past (cox-inhibitors, statins, etc.)

\section{Ontologies and Information Models}

Serialization does not, per se, need understanding. It can easily be separated from ontologies that are describing the semantic of items, objects, actors, etc. Serialization's role is tag every single instances of entities, while the ontologies have the role of defining the meaning of entities. Ontologies for items, services and processes, actors, locations, whatever else to be identified are not at the same level of representation, though this is sometimes confused, such as in Snomed $[22,23]$. In a same manner, there should be no interference with information models, though the same confusion has also been reported [24]. Ontologies and information models should allow supporting entity tracking and serialization, without trying to substitute themselves to this role. To illustrate this point, one can take the numbering of houses in a street. Each house receives number. The number (serialization) is unique within a street. The number does not say anything about the house itself, which would be the ontology.

\section{Persistence in Time}

An important characteristic of healthcare, which is usually not the case in the supply chain, is the very long persistence in time of all elements. Especially when it comes to patient records, or epidemiology, side effects on longterm or drug safety, there is a need for tracking and tracing all along the life of patients and, sometimes, much more. So, for example, an association has been found between the clear cell adenoma of the cervix in young women and the intake of a drug, the stilbestrol, during the pregnancy of their mothers [25-27]. Such a long lag-time over one generation of a side effect of a drug illustrates dramatically the need for a high persistence of traceability. The identification schemes used in healthcare must therefore support persistence in time, which means that once an ID has been used, it can never been used anymore. This is rather different from what is required for usual good supply chain encoding. This issue has been recognized by GS1 and included in its guideline for GTIN allocation. It further corresponds to regulatory expectations, as from the US-FDA.

Epidemiology in public health would be great beneficiaries of a comprehensive and coherent tracking mechanism, especially when it comes to post-market surveillance, such as drug adverse events or implantable devices followup, to name only two examples.

\section{Carriers and Readers}

An important aspect of entity tracking is related to carriers and readers. Using a standardized encoding is not sufficient. Standardized carriers and readers must also be used in order to have the codes be carried by entities, read and stored. There are many sort of possible carriers, such as barcodes, data matrix, RFID, smartcards, etc. There are several standards for each of these carriers, such as barcodes or RFID's. For example, RFID is used on three different frequency ranges, $125 \mathrm{kHz}$, $13.56 \mathrm{GHz}$ and the $900 \mathrm{MHz}$ range, each one having different physical characteristics and, therefore, advantages and disadvantages [28]. Barcodes are the most common used identification carrier and the GS1 standard is very frequent. There are however still problems with reliability of the carriers. The effect of unreadable data carrier is not the same in retail as it is in healthcare. While errors rate of about 1/1'000 are acceptable in the supply chain, they are largely too high in healthcare and reli- 
ability, especially for RFID's. Limitations of RFID in healthcare must still be carefully assessed [29]. Good reliability studies are missing. However, RFID is a very promising technology that will have a significant impact in healthcare [30-32]. Figure 6 illustrates one important advantage of RFID on the example of sticker for chemotherapy infusions. RFID or data matrix replacing barcodes leaves a lot of room on the stocker to print important information for nurses [12].

There is no ideal carrier. The choice of the carrier will depend from many constraints, physical and environmental constraints, but also reliability, robustness and cost. Some carriers, such as active RFID's, can also measure local physical characteristics, for example temperature, which will be important for the logistics. Such an usage is illustrated with blood supply using carriers combining traceability and cold chain controlling. Readers are tightly linked to carriers. Specific readers exist for each type of carriers, and it is important to differentiate between optical carrier and RF carriers. Any optical carriers can be read by image scanners. Unfortunately, there are only few readers that can read several types of radio-frequency carriers. This is an important problem in healthcare where care providers, especially nurses, would be confronted to the handling of several readers to manage all type of carriers, such as RFID's on badges and optical codes on packaging and dispensing units.

\section{Conclusion}

Entity identification and tracking is a major challenge in healthcare. Correct identification of all entities involved, including actors, will allow to increase the supply chain efficiency, to reduce medical errors, to enforce clinical and epidemiological research and governance by leveraging the management understanding of processes involved in healthcare. However, healthcare is a complex sector, far more complex than what is usually the case in consumers' goods production. Numerous factors are involved in this high complexity: technology, persistence in time, serialization, legal frameworks and privacy concerns are major aspects that must be taken into account in order to apply what is well known about traceability in the retail business to healthcare. Most industrial sectors are involved in healthcare, from goods production to high technology, drugs, devices and food supply, making the health sector at the crossroads of many different supply chains, and being itself a service provider and therefore having to deal with huge numbers of items from numerous sources.

The legal framework is slowly moving, at least in specific sectors, such as drugs, mostly as a reaction to counterfeited products. The US federal laws require a certified chain of custody, or pedigree, for pharmaceuticals, which should help to identify all steps between the raw production and the final enduser $[11,33,34]$. The tracing of drugs is increasingly implemented, though it remains marginal compared to the overall use and production of pharmaceuticals. It is remarkable to notice that such type of "pedigree" has been widely implemented in Europe with the General Food Law, which entered into force on 1 January 2005. The new mandatory traceability requirement applies to all food, animal feed, food-producing animals and all types of food chain operators from the farming sector to processing, transport, storage, distribution and retail to the consumer. The "mad-cow" disease crisis which make such political decision possible and which put a considerable public pressure on food industries show the feasibility of such approaches at very large scales even on low cost items.

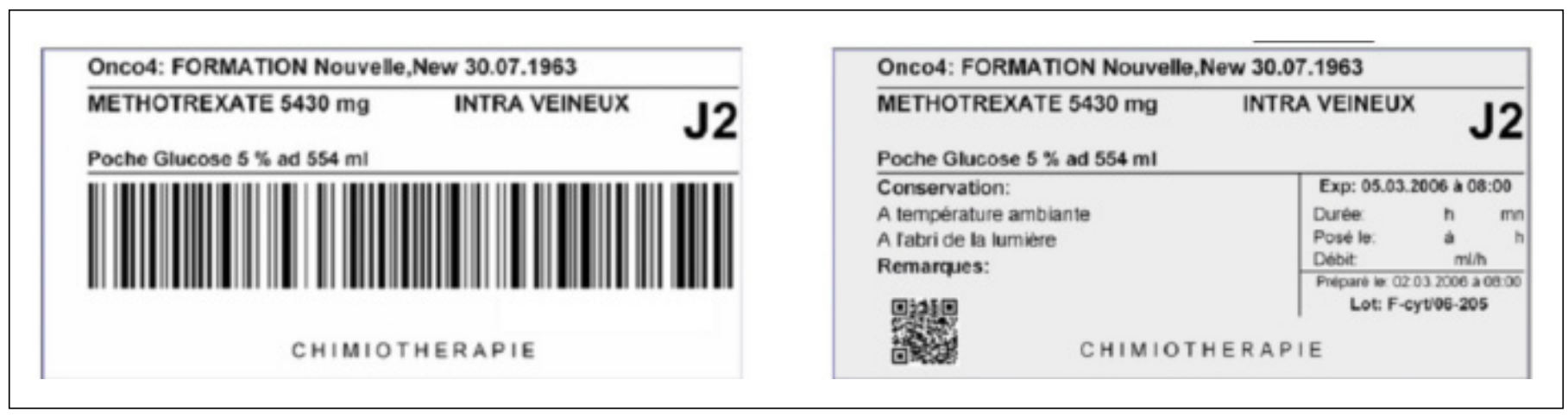

Fig. 6 Chemotherapy stickers: barcode (left) versus RFID or data matrix (right) 
Technical aspects, such as serialization of all items in the supply chain, reliability of carriers and readers, cross linking of GS1 with healthcare standards such as HL7, SNOMED or OpenEHR are still open issues. There are important challenges around semantic and knowledge representation. Solving these issues are mandatory steps to facilitate and allow a wide implementation of traceability in healthcare.

Finally, one must not forget that each progress of traceability is accompanied with a breach in privacy and, potentially, civil liberties. Building a safer healthcare system needs a strong track and trace mechanism, able to rebuild all histories, from their early beginning to late outcomes. This goal must be achieved with the highest level of privacy respect. The future is at stakes [30].

\section{References}

1. Lupien JR. Food quality and safety: traceability and labeling. Crit Rev Food Sci Nutr 2005; 45(2):119-23.

2. Miller JL, Thompson PA, Orabella MM. Forecasting in foodservice: model development, testing, and evaluation. J Am Diet Assoc 1991;91(5):569-74.

3. Rajic A, Waddell LA, Sargeant JM, Read S, Farber J, Firth MJ, et al. An overview of microbial food safety programs in beef, pork, and poultry from farm to processing in Canada. J Food Prot 2007 May;70(5):1286-94.

4. Berg L. Trust in food in the age of mad cow disease: a comparative study of consumers' evaluation of food safety in Belgium, Britain and Norway. Appetite 2004 Feb;42(1):21-32.

5. Pettitt RG. Traceability in the food animal industry and supermarket chains. Rev Sci Tech 2001 Aug;20(2):584-97.

6. Cheek P. Factors impacting the acceptance of traceability in the food supply chain in the United States of America. Rev Sci Tech 2006 Apr;25(1):313-9.

7. Armbruster D, Miller RR. The Joint Committee for Traceability in Laboratory Medicine (JCTLM): A Global Approach to Promote the Standardisation of Clinical Laboratory Test Results. Clin Biochem Rev 2007 Aug;28(3):105-14.

8. Faber JC. Worldwide overview of existing haemovigilance systems. Transfus Apher Sci 2004 Oct;31(2):99-110.

9. Flanagan P. Product traceability and lookback: assuring the integrity of the transfusion process. Dev Biol (Basel) 2007;127:225-33.

10. Gac A, Durand MP. Cold chain - the concept of traceability - practical applications. Bull Acad Natl Med 2001;185(2):301-10.

11. James JS. FDA, companies test RFID tracking to prevent drug counterfeiting. AIDS Treat News 2005 $\operatorname{Dec}(417): 5-8$.

12. Spahni S, Lovis C, Ackermann M, Mach N, Bonnabry P, Geissbuhler A. Securing chemotherapies: fabrication, prescription, administration and complete traceability. Medinfo 2007;12(Pt 2):953-7.

13. Scott MG, Morin S, Hock KG, Seyoum M, Ladenson JH. Establishing a simple and sustainable quality assurance program and clinical chemistry services in Eritrea. Clin Chem 2007 Nov; 53(11):1945-53.

14. Panteghini M. Traceability, reference systems and result comparability. Clin Biochem Rev 2007 Aug;28(3):97-104.

15. Staccini P, Joubert M, Quaranta JF, Fieschi D, Fieschi M. Integration of health care process analysis in the design of a clinical information system: applying to the blood transfusion process. Proc AMIA Symp 2000:824-8.

16. GS1. General specifications 2008. Version 8.1. 2002008 January, 2005

17. ITAC. Report to the President, Revolutionizing Health Care through Information technology. President's Information Technology advisory Committee. June 2004.

18. Auttonberry D. "To err is human"-or is it process? J Miss State Med Assoc 2000;41(2):499.

19. IOM, editor. To Err is Human. Building a safer Health System. National Academy Press; 1999.

20. Kohn LT. To err is human building a safer health system. Washington, D.C.: National Academy Press; 2000.

21. Leape LL, Brennan TA, Laird N, Lawthers AG, Localio AR, Barnes BA, et al. The nature of adverse events in hospitalized patients. Results of the Harvard Medical Practice Study II. N Engl J Med $1991 \mathrm{Feb}$ 7;324(6):377-84.

22. Schulz S, Hanser S, Hahn U, Rogers J. The semantics of procedures and diseases in SNOMED CT. Methods Inf Med 2006;45(4):354-8.

23. Schulz S, Suntisrivaraporn B, Baader F. SNOMED
CT's problem list: ontologists' and logicians' therapy suggestions. Medinfo 2007;12(Pt 1):802-6.

24. Smith B, Ceusters W. HL7 RIM: an incoherent standard. Stud Health Technol Inform 2006; 124:133-8.

25. Hill EC. Clear cell carcinoma of the cervix and vagina in young women. A report of six cases with association of maternal stilbestrol therapy and adenosis of the vagina. Am J Obstet Gynecol 1973 Jun 15;116(4):470-84.

26. Hillemanns HG, Bauknecht T, Simmer H. The stilbestrol-adenosis-carcinoma syndrome. Obstet Gynecol Surv 1979 Nov;34(11):814-7.

27. Ulfelder H. The stilbestrol-adenosis-carcinoma-syndrome. A review. Geburtshilfe Frauenheilkd 1975 May;35(5):329-33.

28. Kabachinski J. An introduction to RFID. Biomed Instrum Technol 2005 Mar-Apr;39(2):131-4.

29. Asamoah AK. Not as easy as it may appear: using radio frequency identification technology to fulfill the Prescription Drug Marketing Act's elusive pedigree requirement. Food Drug Law J 2006;61(2):385418.

30. Boulard G. RFID: promise or peril? It may be easier than ever to track information, but it is causing concerns over privacy and civil liberties. State Legis 2005 Dec;31(10):22-4.

31. Dinh AK. RFID systems in healthcare. Emerging uses and potential issues. J AHIMA 2008 Jan; 79(1):62-3.

32. Fanberg H. The RFID revolution. Mark Health Serv 2004 Fall;24(3):43-4.

33. Prescription Drug Marketing Act Pedigree Requirements; effective date and compliance policy guide; request for comment. Final rule; announcement of effective date; notice of availability; request for comment. Fed Regist 2006 Jun 14;71(114):34249-51.

34. Young D. FDA embraces RFID to protect drug supply. Am J Health Syst Pharm 2004 Dec 15;61(24):2612,5.

\section{Correspondence to:}

Christian Lovis, MD MPH

University Hospitals of Geneva

Service of Medical Informatics, Unit of Clinical Informatics

24, Rue Micheli-du-Crest

1211 Geneva 4

Switzerland

Tel: +41 223726169

E-mail: christian.lovis@hcuge.ch

www.hcuge.ch/sim 